\title{
Electrically Controllable Saturable Absorption in Hybrid Graphene-Silicon Waveguides
}

\author{
Koen Alexander ${ }^{1,2}$, Yingtao $\mathrm{Hu}^{1,2}$, Marianna Pantouvaki ${ }^{3}$, Steven $\mathrm{Brems}^{3}$, Inge \\ Asselberghs $^{3}$, Simon-Pierre Gorza ${ }^{4}$, Cedric Huyghebaert ${ }^{3}$, Joris Van Campenhout ${ }^{3}$, \\ Bart Kuyken $^{1,2}$, Dries Van Thourhout ${ }^{1,2}$ \\ ${ }^{1}$ Photonics Research Group, INTEC, Ghent University-IMEC, Ghent B-9000, Belgium \\ ${ }^{2}$ Center for Nano-and Biophotonics (NB-Photonics), Ghent University, Gent B-9000, Belgium \\ ${ }^{3}$ IMEC, Kapeldreef 75, B-3001 Leuven, Belgium \\ ${ }^{4}$ Service OPERA-photonique, Université libre de Bruxelles (U.L.B.), B-1050 Bruxelles, Belgium
}

Koen.Alexander@intec.ugent.be

\begin{abstract}
Electrical tunability of saturable absorption is demonstrated in a graphene/SOI hybrid waveguide. The saturation modulation depth is tunable between 0 and $2.2 \mathrm{~dB}(\sim 0-40 \%$ decrease of absorption at saturation), with saturation powers between $\sim 1.25-2.5 \mathrm{~W}$.
\end{abstract}

(C) 2014 Optical Society of America

OCIS codes: (130.0130) Integrated Optics, (190.0190) Nonlinear Optics, (320.5390) Picosecond phenomena

\section{Introduction}

Graphene has extraordinary electrical and optical properties, originating from its two-dimensional crystal structure and its linear and gapless band structure [1,2]. One of these well known optical properties is saturable absorption: band filling at high optical powers causes the absorption of graphene to decrease [3]. This phenomenon is exploited in spontaneously mode-locked lasers, taking advantage of the ultra-fast carrier dynamics and broadband absorption of graphene. Early studies have demonstrated mode-locked fiber lasers using an exfoliated monolayer of graphene on a fiber end [3], or a saturable absorber made of graphene flakes dispersed in a polymer [4]. More recently, in-plane saturable absorption of graphene on a silicon waveguide has been demonstrated [5]. This way, the light-graphene interaction can be tailored by changing the waveguide length, and the strong optical confinement and high integration density of silicon-on-insulator (SOI) platforms can lead to compact and cost-efficient integrated devices.

In this work, a graphene-oxide-silicon capacitor structure on top of a silicon rib waveguide is created, so that the Fermi level of graphene can be electrostatically tuned. The voltage dependence of the nonlinear absorption of the structure is investigated and tunable saturable absorption is demonstrated. Since the saturable absorption modulation depth can strongly influence the pulse duration of mode-locked lasers [6], the concepts demonstrated in this work could eventually lead to integrated spontaneously mode-locked lasers with an electrically tunable pulse duration.
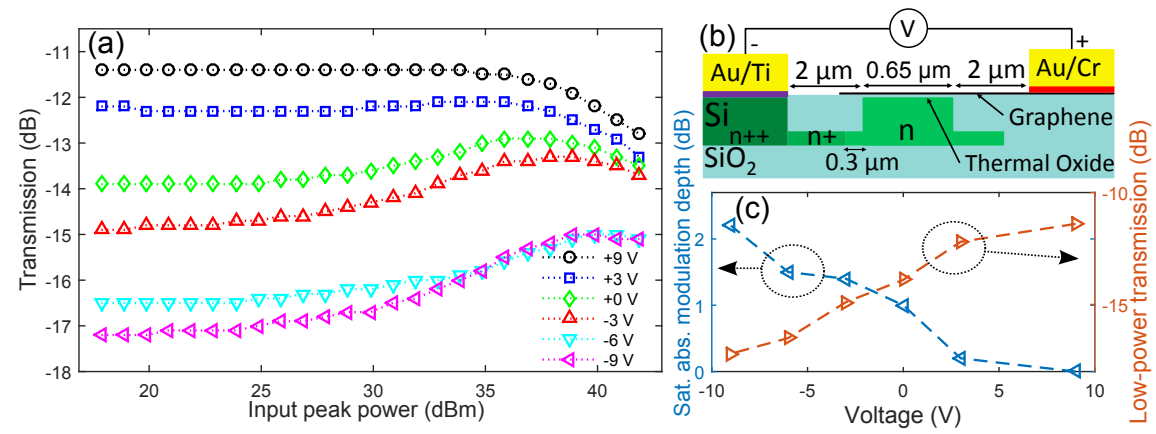

Fig. 1. (a) Transmission of the hybrid graphene/Si waveguide as a function of input peak power (pulse duration: $\sim 8 \mathrm{ps}$, wavelength: $1550 \mathrm{~nm}$ ). (b) Schematic cross section of the hybrid monolayer graphene-Si waveguide structure (doping levels $\mathrm{n}: 10^{18} \mathrm{~cm}^{-3}, \mathrm{n}+: 10^{19} \mathrm{~cm}^{-3}, \mathrm{n}++: 10^{20} \mathrm{~cm}^{-3}$ ). (c) Low-power transmission and saturable absorption modulation depth for different voltages. 


\section{Fabrication and Experimental results}

The cross section of the structure is shown in figure 1(b). A planarized Si rib waveguide (650 nm wide) confines the optical mode. Phosphorous ion implantation was used to lower the electrical resistance of the Si (while keeping the optical loss acceptable, hence the different doping levels) and a $5 \mathrm{~nm}$ thick gate oxide was grown on top of the waveguide. The graphene was grown by chemical vapor deposition (CVD) on a $\mathrm{Si} / \mathrm{SiO}_{2} / \mathrm{Cu}$ substrate and transferred using an elastomer stamp. Both the graphene and the Si waveguide are contacted and by changing the voltage over the graphene/oxide/Si capacitor structure the Fermi level of the graphene can be tuned. These structures can also be used as electro-absorption modulators and more details about this and their fabrication can be found in ref. [7]. The device under study consists of a $150 \mu \mathrm{m}$ long graphene/Si hybrid waveguide. The light is coupled in and out through grating couplers. The couplers and waveguides are optimized for TM light, as the evanescent fields that interact with the graphene are stronger for the TM mode. The coupling loss of the gratings is about 9-10 dB. A PriTel, Inc. FFL1550-10 laser is used to generate $\sim 8$ ps pulses with a repetition rate of $10 \mathrm{MHz}$ with a wavelength of $1550 \mathrm{~nm}$. These pulses are amplified using a Pritel, Inc. HPP-PMFA-20 high peak power Er-doped fiber amplifier and an additional attenuator (JDS FiTel, HA9 Optical attenuator) is used to change the pulse power without significantly changing the pulse shape. Using picosecond pulses and a relatively low repetition rate, the influence of free carriers generated by two-photon absorption in $\mathrm{Si}$ is minimized.

The measured transmission as a function of the input peak power is plotted in figure 1(a), for different voltages. Figure 1(c) shows the low-power transmission and the saturable absorption modulation depth, which is the difference between the peak transmission and the the low-power transmission. At low voltages, the low-power absorption (transmission) is relatively high (low) but shows strong saturation when the optical power increases. This corresponds to a Fermi level close to zero: Pauli blocking is unlikely at low power due to the low electron and hole occupation probabilities at the relevant electronic states (at energies $\pm \hbar \omega / 2$ ). At higher input powers these occupation probabilities can significantly increase, leading to absorption saturation. At high voltages, the Fermi level becomes increasingly larger and the occupation probability at $\hbar \omega / 2$ gradually increases, effectively reducing the number of states available for absorption and thus reducing (increasing) the low-power absorption (transmission). At higher optical powers the changes due to further band-filling will be less dramatic, and the saturable absorption modulation depth will drop. Note that the Fermi level only reaches zero if the voltage is about $-9 \mathrm{~V}$, this is due to spontaneous atmospheric doping of the sample. Furthermore a relatively steep drop in transmission can be observed in the limit of high power. This is caused by strong two-photon absorption in Si. The saturation power $P_{s}$ (optical power at which the absorption change has reached half of its final value) can be estimated to be in the range $31-34 \mathrm{dBm}(\sim 1.25-2.5 \mathrm{~W})$ for all measurements.

\section{Conclusion}

In this paper, electrically tunable saturable absorption in a hybrid graphene/silicon waveguide is to our knowledge for the first time experimentally demonstrated. By tuning the voltage, the saturable absorption modulation depth was tuned between 0 and $2.2 \mathrm{~dB}$ (corresponding to a $40 \%$ decrease in absorption at saturation). Saturation powers ranged between $\sim 1.25-2.5 \mathrm{~W}$. This principle can potentially be used for integrated spontaneously mode-locked lasers with an electrically tunable pulse duration.

\section{References}

1. A. K. Geim, K. S. Novoselov, "The rise of graphene," Nature materials, vol. 6, no. 3, pp. 183-191, 2007.

2. F. Bonaccorso, Z. Sun, T. Hasan, and A. Ferrari, "Graphene photonics and optoelectronics," Nature Photonics, vol. 4, no. 9, pp. 611-622, 2010.

3. Q. Bao, H. Zhang, Y. Wang, Z. Ni, Y. Yan, Z. X. Shen, K. P. Loh, D. Y. Tang, "Atomic-layer graphene as a saturable absorber for ultrafast pulsed lasers," Adv. Funct. Mater., vol. 19, no. 19, 3077-3083 (2009).

4. Z. Sun, T. Hasan, F. Torrisi, D. Popa, G. Privitera, F. Wang, F. Bonaccorso, D. M. Basko, A. C. Ferrari, "Graphene mode-locked ultrafast laser," ACS Nano, vol. 4, no. 2, 803-810, (2010).

5. Z. Shi, C. Y. Wong, Z. Cheng, K. Xu, H. K. Tsang, "In-plane saturable absorption of graphene on silicon waveguides," in Lasers and Electro-Optics Pacific Rim (CLEO-PR), 1-2 (2013).

6. X. He, D. Wang, Z.-B. Liu, "Pulse-width tuning in a passively mode-locked fiber laser with graphene saturable absorber," Photonics Technology Letters, IEEE, vol. 26, 360-363 (2014).

7. Y. T. Hu, M. Pantouvaki, S. Brems, I. Asselberghs, C. Huyghebaert, M. Geisler, C. Alessandri, R. Baets, P. Absil, D. Van Thourhout, and J. Van Campenhout, "Broadband 10gb/s graphene electro-absorption modulator on silicon for chip-level optical interconnects," Electron Devices Meeting (IEDM), 2014 IEEE International. 